\title{
Higher Incidence of Hypospadias in Monochorionic Twins
}

\author{
Remco Visser, ${ }^{1}$ Nienke C. M. Burger, ${ }^{1}$ Erik W. van Zwet, ${ }^{2}$ Yvonne Hilhorst-Hofstee, ${ }^{3}$ Monique C. Haak, ${ }^{4}$ \\ Joop van den Hoek, ${ }^{5}$ Dick Oepkes, ${ }^{4}$ and Enrico Lopriore ${ }^{1}$ \\ ${ }^{1}$ Division of Neonatology, Department of Pediatrics, Leiden University Medical Center, Leiden, The Netherlands \\ ${ }^{2}$ Department of Biostatistics, Leiden University Medical Center, Leiden, The Netherlands \\ ${ }^{3}$ Department of Clinical Genetics, Leiden University Medical Center, Leiden, The Netherlands \\ ${ }^{4}$ Division of Fetal Medicine, Department of Obstetrics, Leiden University Medical Center, Leiden, The Netherlands \\ ${ }^{5}$ Department of Urology, Leiden University Medical Center, Leiden, The Netherlands
}

\begin{abstract}
Background: Hypospadias is associated with twinning. The incidence of hypospadias in monochorionic and dichorionic male twins is, however, yet to be determined. Methods: All medical records of monochorionic and dichorionic twins admitted to our neonatal nursery between January 2004 and August 2013 were reviewed for the presence of hypospadias. Results: A total of 350 monochorionic and 303 dichorionic male twins were included in the study. The incidence of hypospadias in monochorionic and dichorionic groups was $4 \%(14 / 350)$ and $1 \%(3 / 303)(p=.016)$ respectively. In 11 of the 15 twin couples, hypospadias occurred in the twin with the lowest birth weight. The rate of hypospadias in twin infants small-for-gestationalage group was $10 \%(6 / 60)$ compared with $2 \%(11 / 593)$ in the appropriate-for-gestational-age group ( $p=$ .002). In a multivariate analysis, both monochorionicity and small-for-gestational-age were independently associated with hypospadias, odds ratio 4.1 (95\% confidence interval (Cl): 1.1-14.7) and 6.1 (95\% Cl: 2.217.2) respectively. Conclusions: The incidence of hypospadias is four-fold higher in monochorionic twins compared with dichorionic twins. Hypospadias is also independently associated with small-for-gestationalage.
\end{abstract}

Keywords: hypospadia, twins, monochorionic, dichorionic, fetal growth restriction

Hypospadias is one of the most common malformations in males, with an estimated incidence of 3 to 8 per 1,000 newborn males in Europe (Nordenvall et al., 2014; Pierik et al., 2002; Virtanen et al., 2001). In hypospadias, the urethral opening is displaced along the ventral side of the penis. The phenotype is defined by the position of the urethral meatus in anterior (glandular or coronal), middle (penile), and posterior (penoscrotal, scrotal, or perineal) hypospadias (van der Zanden et al., 2012). Hypospadias is the result of an incomplete fusion of the urethral folds, which occurs between the 8 th and 14th week of gestation (Manson \& Carr, 2003). The earlier the fusion fails in the embryonic period, the more severe the type of hypospadias (i.e., posterior hypospadias).

The etiology of isolated hypospadias is considered to be multifactorial: genetic, endocrine, environmental, and clinical factors have been implicated (van der Zanden et al., 2012). A family history of hypospadias increases the risk by seven- to 10-fold, suggesting an important genetic component (Brouwers et al., 2007; Fredell et al., 2002; van Rooij et al., 2013).
Association between low birth weight and hypospadias is well established (Fredell et al., 2002; Weidner et al., 1999). In particular, being small-for-gestational-age (SGA) has been shown to be an important risk factor for hypospadias (Akre et al., 1999; Ghirri et al., 2009; Jensen et al., 2012; Nordenvall et al., 2014). Studies suggest that intra-uterine growth restriction (IUGR) in itself is a determining risk factor for hypospadias rather than the absolute birth weight (Hussain et al., 2002; Jensen et al., 2012; Yinon et al., 2010).

Furthermore, twinning is a risk factor consistently associated with hypospadias (Brouwers et al., 2007; Jensen et al., 2012; Nordenvall et al., 2014; van Rooij et al., 2013). In a large case-control study about structural birth defects in

RECEIVED 17 February 2015; ACCEPTED 6 July 2015. First published online 14 August 2015.

ADDRESS FOR CORRESPONDENCE: Dr E. Lopriore, MD, PhD, Division of Neonatology, Department of Pediatrics, J6-S, Leiden University Medical Center, PO Box 9600, 2300 RC, Leiden, The Netherlands. E-mail: e.lopriore@lumc.nl 
TABLE 1

Baseline Characteristics of MC and DC Twin Groups

\begin{tabular}{llll}
\hline Characteristics & $\mathrm{MC}(n=350)$ & $\mathrm{DC}(n=303)$ & $p$-value \\
\hline Mean birth weight $(\mathrm{g})$ & $1759 \pm 583$ & $1845 \pm 653$ & .08 \\
Mean gestational age at birth (weeks) & $32.0 \pm 3.0$ & $32.1 \pm 3.6$ & .7 \\
Small for gestational age $-n(\%)$ & $42(12 \%)$ & $18(6 \%)$ & .01 \\
\hline
\end{tabular}

twins, hypospadias occurred more often in twins compared with singletons (adjusted odds ratio (OR) 2.1 [95\% CI: 1.53.1]; Rider et al., 2013). In addition, hypospadias in birth weight discordant twins is more frequently found in the twin with the lowest birth weight (Chambers et al., 2006; Fredell et al., 1998). Since this was determined in monozygotic twin pairs, genetic factors were excluded, suggesting the importance of environmental etiological risk factors for hypospadias in these twins (Fredell et al., 1998). However, in the study by Fredell et al. (1998) no information was provided about chorionicity. To date, the rate of hypospadias in monochorionic (MC) and dichorionic (DC) twins has not been reported in literature. Therefore, the objective of this study was to determine the incidence of hypospadias in $\mathrm{MC}$ and DC male twins. Furthermore, the possible correlation between hypospadias and SGA-discordant twins was investigated.

\section{Material and Methods}

All MC and DC male twins admitted to the neonatal intensive care unit at the Leiden University Medical Centre, the Netherlands, between January 2004 and August 2013 were included in this study. Data were extracted from the hospital's patient database, and all collected data were anonymized. The following perinatal data were recorded: birth weight, gestational age at birth, SGA (defined as a birth weight of $<10$ th centile for gestational age according to Dutch references; Kloosterman, 1969), and the presence and type of hypospadias. We excluded cases with major congenital anomalies. Chorionicity was determined by prenatal ultrasound, and after birth by macroscopic examination of the placenta and the inter-twin membrane.

\section{Statistics}

Results from MC and DC twin groups were compared using an independent $t$-test for categorical data and the chisquare test for dichotomous data. Univariate logistic regression analysis was performed to investigate the possible relationship between hypospadias, type of chorionicity, and SGA. The multivariate logistic regression model included all variables that showed significant association in univariate analysis. Results are expressed as OR with $95 \%$ confidence interval (CI). All analyses were conducted using the generalized estimated equation module to account for the effect that observations within twins are not independent. A $p$-value of $<.05$ was considered to indicate statistical sig-
TABLE 2

Hypospadias Phenotype

\begin{tabular}{llll}
\hline & MC $(n=350)$ & DC $(n=303)$ & $p$-value \\
\hline Hypospadias (\%) & $14(4 \%)$ & $3(1 \%)$ & .016 \\
Type of hypospadias & & & \\
$\quad$ Glandular & 9 & 1 & \\
Coronal & 2 & 2 & \\
$\quad$ Unspecified & 3 & 0 & \\
Hypospadias in both twins & $4 / 14$ & $0 / 3$ & \\
\hline
\end{tabular}

nificance. Statistical data were analyzed using SPSS statistic version 20.0 (SPSS Inc. Chicago, IL).

\section{Results}

Between January 2004 and August 2013, a total of 360 MC and 306 DC male twins were admitted to our neonatal nursery. Thirteen patients were excluded because of major congenital birth defects. Therefore, analysis was performed, including $350 \mathrm{MC}$ and $303 \mathrm{DC}$ male twins. Baseline characteristics of the study population are presented in Table 1.

A total of 17 patients with hypospadias were identified in our study cohort: 14 of $350(4 \%)$ in the MC group and 3 of $303(1 \%)$ in the DC group $(p=.016)$. Table 2 shows different phenotypes of hypospadias in both groups. In two MC twin pairs, both twin brothers had hypospadias, while the other 10 twin pairs were discordant for hypospadias. In nine of the 12 (75\%) MC twin pairs, the twin with the lowest birth weight had hypospadias. In one pair, the birth weight was identical in both infants. In DC twins, all hypospadias occurred in the same-sex twin pairs. All three DC twins were discordant for hypospadias, with two having a lower birth weight than their unaffected brother and one having higher birth weight. Overall, in 11 of the $15(73 \%)$ twin pairs, the twin with the lowest birth weight had hypospadias.

In the MC group, in total, 42 males (12\%) were born SGA and $18(6 \%)$ in the DC group $(p=.008)$. The rate of hypospadias in SGA and non-SGA groups was 10\% (6/60) and $2 \%(11 / 593 ; p=.002)$ respectively. Of the $14 \mathrm{MC}$ twins with hypospadias, six were SGA at birth, whereas none of the DC twins with hypospadias was born SGA. In a multivariate logistic regression model (see Table 3), monochorionicity was found to be an independent risk factor for hypospadias (OR 4.1, 95\% CI: 1.1-14.7, $p=.032$ ) as well as SGA (OR $6.1,95 \%$ CI: $2.2-17.2, p=.001)$. 


\section{TABLE 3}

Analysis of Potential Risk Factors for Hypospadias

\begin{tabular}{lccllll}
\hline Risk factors & Hypospadias, yes $(n=17)$ & Hypospadias, no $(n=636)$ & $p$ & Univariate OR $(95 \%$ Cl) & $p$ & Multivariate OR (95\% Cl) \\
\hline MC & $14 / 350(4 \%)$ & $336 / 350(96 \%)$ & .016 & $4.2(1.1-18.4)$ & .032 & $4.1(1.1-14.7)$ \\
DC & $3 / 303(1 \%)$ & $300 / 303(99 \%)$ & & & \\
SGA, yes & $6 / 60(10 \%)$ & $54 / 60(90 \%)$ & .002 & $5.9(1.8-18.6)$ & .001 & $6.1(2.2-17.2)$ \\
SGA, no & $11 / 593(2 \%)$ & $582 / 593(98 \%)$ & & & \\
\hline
\end{tabular}

\section{Discussion}

Hypospadias is known to occur more frequently in twins in comparison with singletons (Nordenvall et al., 2014; Rider et al., 2013). This is the first study showing that the incidence of hypospadias among $\mathrm{MC}$ twins is significantly higher than in DC twins ( $4 \%$ vs. $1 \%$ respectively). Our findings are in line with a previous study describing approximately a two-fold higher incidence of congenital anomalies in MC compared with DC twins (Glinianaia et al., 2008). In that specific study, hypospadias, however, was not reported. The association between monozygosity and hypospadias had been previously suggested by Fredell et al. (2002). In their study, the zygosity status could, however, only be established in $83 \%$ of the twins $(33 / 40 ; 67 \%$ monozygotic and $33 \%$ dizygotic), and chorionicity was not determined. In a different study, the rate of hypospadias was similar for the same-sex twins (a mixture of monozygotic and dizygotic twins) and the opposite-sex twins (purely dizygotic; Rider et al., 2013). Once more, zygosity and chorionic status was not determined. Since MC twins are by definition monozygotic, $82 \%$ (14/17) of twins with hypospadias in our study were thus monozygotic. For our DC group, data about zygosity were not available; however, in general, around 90\% of the DC group is dizygotic and only $10 \%$ is monozygotic (Cameron, 1968). Our data, showing an increased risk of hypospadia in MC twins, is clinically relevant since chorionicity in contrast to zygosity is easily determined during pregnancy with antenatal ultrasound in the first trimester.

In our study, in nine of the $10 \mathrm{MC}$ twin pairs discordant for hypospadias, it occurred only in the smaller twin. This was also the case for two of the three cases in the DC twin pairs. A previous study had also shown that in 16 of the 18 monozygotic twin pairs the twin with the lowest birth weight indeed had hypospadias (Fredell et al., 1998). In addition, in three MC and two DC twin pairs, all hypospadias were reported in the smaller pair of discordant IUGR twins (Yinon et al., 2010). Chambers et al. (2006) described an association of IUGR and hypospadias in like-sex twins. However, zygosity or chorionicity status could not be determined. Furthermore, in singletons, hypospadias occurs more frequently in SGA infants (Gatti et al., 2001; Hussain et al., 2002). This correlation between hypospadias and SGA may suggest an association between placental insufficiency and abnormal genital development. Hypothetically, nutrients and gonadotropins may be insufficient for the normal development of the two pairs of male gonads in twin preg- nancies due to inadequate placentation or unequal placental sharing (Fredell et al., 1998). The smaller twin would be more sensitive to relative deficit in hormones and would therefore be at increased risk of developing hypospadias.

Interestingly, the incidence of hypospadias in MC twins compared with DC twins cannot be explained by a higher incidence of SGA in MC twins because SGA was identified as an independent risk factor for hypospadias in our study. It could be that the above-mentioned environmental nutrient deficiency plays a larger role in MC twins due to sharing of single placenta. Furthermore, it can be speculated that a possible etiological explanation might lie in a factor or mechanism at the timing of monozygotic twinning event. For example, post-zygotic unequal distribution of blastomeres to both embryos might result in structural malformations and growth deficiency (Machin, 1996).

Care should be taken when interpreting the results of our study due to some limitations. First, the retrospective design is dependent on charted data. Second, an ascertainment bias might have occurred, since only admitted infants to our tertiary level neonatal intensive care unit are recorded in the database. This is, for example, the cause for the lower than expected gestational age for twins. However, the gestational age at birth was similar for both $\mathrm{MC}$ and DC groups (32.0 vs. 32.1 weeks respectively) and this should not have influenced our results. Lastly, the study comprises a relative small sample size, hence a small number of hypospadias. Nevertheless, with regard to the studies described in twins in literature, including data about zygosity and/or chorionicity, our number is relatively large.

In conclusion, $\mathrm{MC}$ and DC twins have an increased risk for hypospadias with the incidence being four-fold higher in MC twins than in DC twins. Monochorionicity and SGA were identified to be independently associated with hypospadias.

\section{References}

Akre, O., Lipworth, L., Cnattingius, S., Sparen, P., \& Ekbom, A. (1999). Risk factor patterns for cryptorchidism and hypospadias. Epidemiology, 10, 364-369.

Brouwers, M. M., Feitz, W. F., Roelofs, L. A., Kiemeney, L. A., de Gier, R. P., \& Roeleveld, N. (2007). Risk factors for hypospadias. European Journal of Pediatrics, 166, 671-678.

Cameron, A. H. (1968). The Birmingham twin survey. Proceedings of the Royal Society of Medicine, 61, 229-234. 
Chambers, C. D., Castilla, E. E., Orioli, I., \& Jones, K. L. (2006). Intrauterine growth restriction in like-sex twins discordant for structural defects. Birth Defects Research Part A: Clinical and Molecular Teratology, 76, 246-248.

Fredell, L., Kockum, I., Hansson, E., Holmner, S., Lundquist, L., Lackgren, G., ... Nordenskjold, A. (2002). Heredity of hypospadias and the significance of low birth weight. Journal of Urology, 167, 1423-1427.

Fredell, L., Lichtenstein, P., Pedersen, N. L., Svensson, J., \& Nordenskjold, A. (1998). Hypospadias is related to birth weight in discordant monozygotic twins. Journal of Urology, 160, 2197-2199.

Gatti, J. M., Kirsch, A. J., Troyer, W. A., Perez-Brayfield, M. R., Smith, E. A., \& Scherz, H. C. (2001). Increased incidence of hypospadias in small-for-gestational age infants in a neonatal intensive-care unit. BJU International, 87, 548-550.

Ghirri, P., Scaramuzzo, R. T., Bertelloni, S., Pardi, D., Celandroni, A., Cocchi, G., ... Boldrini, A. (2009). Prevalence of hypospadias in Italy according to severity, gestational age, and birthweight: An epidemiological study. Italian Journal of Pediatrics, 35, 18.

Glinianaia, S. V., Rankin, J., \& Wright, C. (2008). Congenital anomalies in twins: A register-based study. Human Reproduction, 23, 1306-1311.

Hussain, N., Chaghtai, A., Herndon, C. D., Herson, V. C., Rosenkrantz, T. S., \& McKenna, P. H. (2002). Hypospadias and early gestation growth restriction in infants. Pediatrics, 109, 473-478.

Jensen, M. S., Wilcox, A. J., Olsen, J., Bonde, J. P., Thulstrup, A. M., Ramlau-Hansen, C. H., ... Henriksen, T. B. (2012). Cryptorchidism and hypospadias in a cohort of 934,538 Danish boys: The role of birth weight, gestational age, body dimensions, and fetal growth. American Journal of Epidemiology, 175, 917-925.

Kloosterman, G. J. (1969). Intrauterine growth and intrauterine growth curves. Maandschrift Voor Kindergeneeskunde, 37, 209-225.

Machin, G. A. (1996). Some causes of genotypic and phenotypic discordance in monozygotic twin pairs. American Journal of Medical Genetics, 61, 216-228.
Manson, J. M., \& Carr, M. C. (2003). Molecular epidemiology of hypospadias: Review of genetic and environmental risk factors. Birth Defects Research, Part A: Clinical and Molecular Teratology, 67, 825-836.

Nordenvall, A. S., Frisen, L., Nordenstrom, A., Lichtenstein, P., \& Nordenskjold, A. (2014). Population-based nationwide study of hypospadias in Sweden, 1973 to 2009: Incidence and risk factors. Journal of Urology, 191, 783789.

Pierik, F. H., Burdorf, A., Nijman, J. M., de Muinck KeizerSchrama, S. M., Juttmann, R. E., \& Weber, R. F. (2002). A high hypospadias rate in the Netherlands. Human Reproduction, 17, 1112-1115.

Rider, R. A., Stevenson, D. A., Rinsky, J. E., \& Feldkamp, M. L. (2013). Association of twinning and maternal age with major structural birth defects in Utah, 1999 to 2008. Birth Defects Research, Part A: Clinical and Molecular Teratology, 97, 554-563.

van der Zanden, L. F., van Rooij, I. A., Feitz, W. F., Franke, B., Knoers, N. V., \& Roeleveld, N. (2012). Aetiology of hypospadias: A systematic review of genes and environment. Human Reproduction Update, 18, 260-283.

van Rooij, I. A., van der Zanden, L. F., Brouwers, M. M., Knoers, N. V., Feitz, W. F., \& Roeleveld, N. (2013). Risk factors for different phenotypes of hypospadias: Results from a Dutch case-control study. BJU International, 112, 121128.

Virtanen, H. E., Kaleva, M., Haavisto, A. M., Schmidt, I. M., Chellakooty, M., Main, K. M., ... Toppari, J. (2001). The birth rate of hypospadias in the Turku area in Finland. APMIS, 109, 96-100.

Weidner, I. S., Moller, H., Jensen, T. K., \& Skakkebaek, N. E. (1999). Risk factors for cryptorchidism and hypospadias. Journal of Urology, 161, 1606-1609.

Yinon, Y., Kingdom, J. C., Proctor, L. K., Kelly, E. N., Salle, J. L., Wherrett, D., ... Chitayat, D. (2010). Hypospadias in males with intrauterine growth restriction due to placental insufficiency: The placental role in the embryogenesis of male external genitalia. American Journal of Medical Genetics Part A, 152A, 75-83. 